\title{
MODELLING OF IMPULSE LOAD INFLUENCE ON THE STRESS STATE OF FOAM MATERIALS WITH POSITIVE AND NEGATIVE POISSON'S RATIO
}

\author{
Heorhiy SULYM*, Olena MIKULICH**, Vasyl' SHVABYUK ${ }^{* *}$ \\ *Faculty of Mechanical Engineering, Department of Mechanics and Applied Computer Science, \\ Bialystok University of Technology, ul. Wiejska 45C, 15-351 Bialystok, Poland \\ *Faculty of Architecture, Construction and Design, Department of Applied Mathematics and Mechanics, \\ Lutsk National Technical University, 75 Lvivska st., Lutsk, 43018, Ukraine \\ shyprao@gmail.com, h.sulym@pb.edu.pl,v.shvabyuk@gmail.com
}

received 3 December 2019, revised 21 May 2020, accepted 26 May 2020

\begin{abstract}
The influence of impulse load applied for different duration on the distribution of normalised dynamic radial stresses in positive and negative Poisson's ratio medium was investigated in this study. For solving the non-stationary problem in the case of plane deformation for structurally inhomogeneous materials, the model of Cosserat continuum was applied. This model enables accounting for the influence of shear-rotation deformation of micro-particles of the medium. In the framework of Cosserat elasticity, on applying the Fourier transforms for time variable and developing the boundary integral equation method, solving of the non-stationary problem reduces to the system of singular integral equations, where the components that determine the influence of shear-rotation deformations are selected. The numerical calculations were performed for the foam medium with positive and negative Poisson's ratio for different values of time duration of impulse. Developed approach can be used to predict the mechanical behaviour of foam auxetic materials obtained at different values of a volumetric compression ratio under the action of time variable load based on analysis of the distribution of radial stresses in foam medium.
\end{abstract}

Key words: Stress state, Foam, Poisson's ratio, Cosserat elasticity

\section{INTRODUCTION}

In recent years, the interest of scientists has grown to the creation of new materials, which have additional functional capabilities. These additional functional capabilities are wider than the properties which are defined by the composition of the material. Such materials are called smart materials. Expansion of their functional capabilities is based on the peculiarities of their internal structure. These materials also include the materials with negative Poisson's ratio $v$ (Scarpa et al., 2016; Brighenti, 2014). Such materials are called auxetics (Evans, 1991). Under the action of stretching force, they become thicker perpendicular to the applied force.

Reviews of auxetic materials and the possibility of their application were made by Carneiro et al. (2013), Novak et al. (2016), Ren et al. (2018) and others.

Developing the technology for creation of materials with a negative Poisson's ratio is important for using them in sports applications (Duncan et al., 2018) and other aspects (Naik et al., 2019).

Thus, in the work of Lakes (1991), the method of creating synthetic materials with negative Poisson's ratio was described. This method was based on triaxial compression and heat treatment of as-received foam for converting it to negative Poisson's ratio foam. An alternative method converting open-cell polyurethane foam into auxetic foam was based on using chemo-mechanical process (Underhill, 2017).

The researches of Lakes (2016), Rueger et al. (2016), Li et al.
(2016) and others were dedicated to the investigation and comparison of elastic characteristics of foam materials with positive and negative Poisson's ratio. These experiments were performed in the framework of Cosserat elasticity. Applying the model of the moment theory of elasticity, we can account for the influence of material microstructure through consideration of shear-rotation deformation of micro-particles of a medium.

The results of the research performed (Lakes, 2016; Rueger et al., 2016; Li et al., 2016, etc.) confirm that the refined models of continuous medium mechanics should be used for investigation of the deformation processes in structurally inhomogeneous materials since the obtained results cannot be correctly described using the equations of the classical theory of elasticity. However, accounting for the influence of shear-rotation deformations leads to the complication of motion equation of the micropolar medium and obtaining the solutions with corresponding problems (Sulym et al., 2018).

A lot of papers investigated the dynamic behaviour of auxetics with different internal structures using computational analysis. In the work of Zang et al. (2014), the in-plane dynamic crushing behaviour of honeycomb auxetics with various cell wall angles was studied using dynamic finite element simulation. Strek et al. (2019) performed computational analysis of the dynamic behaviour of a three-layered sandwich beam with a metal foam core.

Besides, comparing the stress distribution in the initial foam material and auxetic foam obtained based on numerical modelling of the influence of time variable load on the stress state of these materials has a practical interest too. These numerical calculations must be performed in the framework of Cosserat elasticity. 
Sylum et al. (2018) developed the method of investigating the stress state of structurally inhomogeneous materials in Cosserat elasticity. This method was based on the application of Fourier transforms of time variable and development of boundary integral equation method in the framework moment theory of elasticity.

Therefore, the aim of the work is comparison of the dynamic stress state of foam media with positive and negative Poisson's ratios under the action of impulse load which is applied to the boundary of tunnel cavity.

\section{STATEMENT AND SOLUTION OF THE PROBLEM}

\subsection{Statement of the problem}

Consider a foam medium with the tunnel cavity of constant cross section (Sulym et al., 2018). To the boundary of the cavity, non-stationary load is applied in the normal direction. Denote the boundary of a cross section of the cavity by $L$ (Fig. 1). The centre of gravity is placed at the origin of a Cartesian coordinate system $O x_{1} x_{2}$.

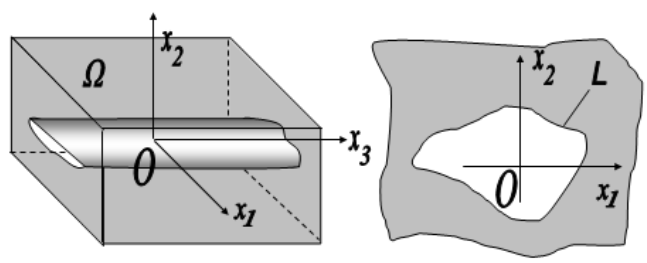

Fig. 1. 3D full geometry and 2D plane geometry of the problem

The problem consists in the determination and comparison of the dynamic stresses at negative and positive Poisson's ratio media with the tunnel cavity under the action of impulse load applied for different duration in a direction normal to the boundary of cavity.

The boundary conditions of the problem are written as (Sulym et al., 2018):

$\left.\sigma_{n}\right|_{L}=\sigma_{0} P(t),\left.\tau_{s n}\right|_{L}=0,\left.m_{n}\right|_{L}=0$,

where $\sigma_{0}$ is a constant and $P(t)$ is a known function which describes the change of load intensity over time.

\subsection{Solution of the problem}

If applying load in a direction normal to the boundary of cavity one can solving of $3 \mathrm{D}$ problem convert to solving $2 \mathrm{D}$ problem in case of plane deformation.

For the investigation of the stress state of the micropolar medium under the action of time variable load, the motion equations of Cosserat elasticity are used (Nowacki, 1974):

$\sigma_{j i, j}+X_{i}=\rho \ddot{u}_{i}$,

$\epsilon_{k i j} \sigma_{i j}+m_{j k, j}+Y_{k}=J \ddot{\phi}_{i}$,

where $\sigma_{j i}$ is the force stress, $m_{j i}$ is the couple stress, $\rho$ is the material density, $\mathbf{X}=\left\{X_{i}\right\}$ is the mass forces vector, $\mathbf{Y}=\left\{Y_{i}\right\}$ is the couple forces vector, $J$ is the inertia of unit volume rotation, $\epsilon_{k l m}$ is the permutation symbol, $\boldsymbol{u}=\left\{u_{i}\right\}$ is the displacement vector and $\phi=\left\{\phi_{k}\right\}$ is the rotation vector. Functions $u_{i}$ and $\phi_{k}$ are continuous functions.

Here and further, the Einstein summation convention is used. A comma at subscript denotes differentiation with respect to a coordinate indexed after the comma, i.e. $u_{j, i}=\partial u_{j} / \partial x_{i}$. Under the condition of plane, strain indices vary from 1 to 2 , and $k=3$.

The dependencies for determining force and couple stresses are written as (Nowacki, 1974):

$\sigma_{j i}=(\mu+\alpha) \gamma_{j i}+(\mu-\alpha) \gamma_{i j}+\lambda \gamma_{k k} \delta_{i j}$,

$m_{j i}=(\gamma+\varepsilon) \kappa_{j i}+(\gamma-\varepsilon) \kappa_{i j}+\beta \kappa_{k k} \delta_{i j}$,

where $\alpha, \beta, \gamma$ and $\kappa$ are the elastic constants required to describe an isotropic constrained Cosserat elastic solid, $\lambda$ and $\mu$ are Lame parameters, $\gamma_{i j}=u_{i, j}-\epsilon_{k j i} \phi_{k}$ is the asymmetric deformation tensor and $\kappa_{i j}=\phi_{i, j}$ is the torsion bending tensor.

For solving the non-stationary problem of a dynamic stress state investigation in auxetic foam materials, Fourier transforms is used:

$\hat{f}(\boldsymbol{x}, \omega)=\int_{-\infty}^{\infty} f(\boldsymbol{x}, t) e^{-i \omega t} d t$,

where $i=\sqrt{-1}$ and $\omega$ is the frequency.

Applying Fourier transforms (5) to the motion equations (2) and (3), we obtain the equations which are equivalent to the equations of time-harmonic motion with cyclic frequency $\omega$. Applying the weighted residual approach and collocation method (Vincent et al., 1994) to the obtained equations, the representations of displacement and microrotation transforms for the case of absence of concentrated forces are written as (Sulym et al., 2018):

$\hat{u}_{i}=\int_{L} p_{j} \cdot U_{i j}^{*} d L+\int_{L} m_{k} \cdot \Phi_{k j}^{*} d L$,

$\hat{\phi}_{k}=\int_{L} p_{j} \cdot U_{k j}^{* *} d L+\int_{L} m_{k} \cdot \Phi_{k k}^{* *} d L$,

where $U_{i j}^{*}, U_{k j}^{* *}, \Phi_{k j}^{*}$ and $\Phi_{k k}^{* *}$ are the fundamental functions for displacements and microrotations and $p_{j}, m_{k}$ are unknown functions (Sulym et al., 2018). Sulym et al. (2018) showed that the obtained representations for the fundamental functions for displacements and microrotations $U_{i j}^{*}, U_{k j}^{* *}, \Phi_{k j}^{*}$ and $\Phi_{k k}^{* *}$ contain the components which correspond of the classical theory of elasticity and the components which are obtained in the framework of Cosserat elasticity.

In the field of Fourier transforms, we substitute the displacement and microrotation representations (6) and (7) with the formulas for determining force and couple stresses (4), thus satisfying the Fourier transforms of boundary condition (1).

Therefore, for applying the approach which was developed by Mikulich et al. (2017), we write integral functions in a complex form. Selecting the irregular components and using the formulas of Sokhotski-Plemelj for the limiting boundary values of the Cauchy integrals, we obtain the integral equations for determining the unknown on the boundary functions $p_{1}, p_{2} m_{3}$ in the form:

$\frac{\operatorname{Re}(q)}{2}+P V \int_{L}\left(f_{1}(\zeta, \bar{\zeta}, z, \bar{z}) \cdot q \cdot d t+f_{2}(\zeta, \bar{\zeta}, z, \bar{z}) \cdot \bar{q} \cdot d \bar{t}+\right.$

$\left.f_{3}(\zeta, \bar{\zeta}, z, \bar{z}) \cdot m_{3} \cdot d L\right)=\sigma_{0} \hat{P}(\omega)$,

$\frac{\operatorname{Im}(q)}{2} \vartheta_{1}+P V \int_{L}\left(g_{1}(\zeta, \bar{\zeta}, z, \bar{z}) \cdot q \cdot d t+g_{2}(\zeta, \bar{\zeta}, z, \bar{z}) \cdot \bar{q} \cdot\right.$

$\left.d \bar{t}+g_{3}(\zeta, \bar{\zeta}, z, \bar{z}) \cdot m_{3} \cdot d L\right)=0$, 
$\frac{m_{3}}{2}+P V \int_{L}\left(G_{1}(\zeta, \bar{\zeta}, z, \bar{z}) \cdot q \cdot d t+G_{2}(\zeta, \bar{\zeta}, z, \bar{z}) \cdot \bar{q} \cdot d \bar{t}+\right.$

$\left.G_{3}(\zeta, \bar{\zeta}, z, \bar{z}) \cdot m_{3} \cdot d L\right)=0$,

where $f_{l}(\bullet), g_{l}(\bullet)$ and $G_{l}(\bullet)$ are the known complex functions (Sulym et al., 2018), $\vartheta_{1}=\left(1-\left(\frac{\alpha}{\mu}\right)^{2}\right)$ is a constant, and $\zeta=x_{1}+i \cdot x_{1}, \quad z=x_{1}^{0}+i \cdot x_{2}^{0}, \quad p \cdot d L=i \cdot q \cdot d t, \quad p=$ $p_{1}+i \cdot p_{2}$. Here, the integrals are understood in the sense of Cauchy principal value.

The system of integral equations (8)-(10) is solved numerically by applying the method which is based on the mechanical quadrature method and collocation method (Mikulich et al., 2017).

For determining the Fourier transform of radial stresses, the integral representation is used (Sulym et al., 2018):

$\widehat{\sigma}_{r}=P V \int_{L}\left(h_{4}\left(\boldsymbol{x}, \boldsymbol{x}^{0}\right) \cdot p_{1}+h_{5}\left(\boldsymbol{x}, \boldsymbol{x}^{0}\right) \cdot p_{2}+\right.$

where $h_{k}(\bullet)$ are known functions.

On the basis of the solutions of a system of integral equations, the values of dynamic stresses are obtained in a numerical form. For calculation of originals of the dynamic hoop and radial stresses, the modified discrete Fourier transform is used (Sulym et al., 2018):

$$
\begin{aligned}
& \sigma_{r}\left(t_{k}\right)=\frac{2}{T} \operatorname{Re} \sum_{n=0}^{K-1}\left(\widehat{\sigma}_{r}\left(\omega_{n}\right) \exp \left(2 \pi i \frac{n \cdot k}{K}\right)-\right. \\
& \left.-\sum_{n=0}^{K-1} \widehat{\sigma}_{r}\left(\omega_{n}\right)\right),
\end{aligned}
$$

where $K$ is the number of elements of selection, $\omega_{n}=2 \pi n / t$ is the frequency and $t_{k}=k \cdot t / K, k=0 \ldots K-1$ is time.

\section{NUMERICAL CALCULATION OF STRESSES}

Let us study the influence of time duration of impulse load on the distribution of radial stresses in positive and negative Poisson's ratio polymer foam media with a tunnel cavity of circular cross section.

For numerical calculations, change of the load intensity over time is chosen in the form of weak shock impulse (Mikulich et al., 2017):

$P(t)=p_{*} \bar{t}^{n_{*}} e^{-\alpha_{*} \bar{t}}, \bar{t}>0, n_{*} \geq 0$,

where $p_{*}, n_{*}$ and $\alpha_{*}$ are constants, $\bar{t}=\frac{t \cdot c_{l}}{a}$ is the time parameter, $c_{l}=\sqrt{\frac{\lambda+2 \mu}{\rho}}$ is the speed of the expansion wave and $a$ is the character size (for the case of tunnel cavity of circular cross section, $a=R$, where $R$ is the radius of cavity).

The numerical calculations are performed for the foam material with negative and positive Poisson's ratio foam. The values of elastic characteristics of positive and negative foam materials are used with the work of Rueger et al. (2016). Here auxetic foam material was obtained under triaxial compression and heat treatment of positive Poisson's ratio foam.

The internal structure of positive Poisson's ratio foam is modelled as the complex of honeycomb with average cell size $h$ (Fig. 2a), whereas the internal structure of the negative Poisson's ratio foam, obtained via triaxial compression, is modelled as the complex of re-entrant cells (Fig. 2b) (Novak et al., 2016; Ren et al., 2018).

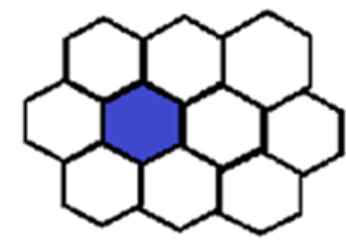

a

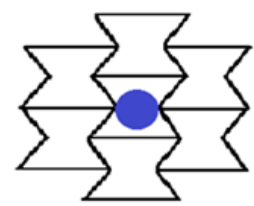

b
Fig. 2. 2D plane model of the foam material with positive (a) and negative (b) Poisson's ratio

In Rueger et al.'s (2016) study for positive Poisson's ratio foam, values of the physical characteristics were obtained: the density of foam was $\rho=30 \frac{\mathrm{kg}}{\mathrm{m}^{3}}$, the shear modulus was $\mathrm{G}=45 \mathrm{kPa}$, length characteristics in Cosserat elasticity were $\ell_{\mathrm{t}}=2.1 \mathrm{~mm}$ and $\ell_{\mathrm{b}}=9 \mathrm{~mm}$ and Poisson's ratio was $v=0.3$. The inertia of unit volume rotation of honeycomb elements in case of $\mathrm{h}=1.2 \mathrm{~mm}$ was $\mathrm{J}=72.4 \cdot \times 10^{-6} \frac{\mathrm{kg}}{\mathrm{m}}$.

For a foam material with negative Poisson's ratio with a volumetric compression ratio 3.2 , the value of an acute angle of a reentrant element was $58.43^{\circ}$. This value is obtained without accounting for bending deformations of the edges of the cells. The inertia of unit volume rotation of re-entrant elements was $\mathrm{J}=$ $29.34 \cdot \times 10^{-6} \frac{\mathrm{kg}}{\mathrm{m}}$. For auxetic foam, the physical characteristics of the obtained material were as follows: the density of foam specimens was $\rho=96 \frac{\mathrm{kg}}{\mathrm{m}^{3}}$, the shear modulus was $\mathrm{G}=$ $16 \mathrm{kPa}$, characteristics of length in Cosserat elasticity were $\ell_{\mathrm{t}}=2.3 \mathrm{~mm}$ and $\ell_{\mathrm{b}}=3.9 \mathrm{~mm}$ and negative Poisson's ratio was $v=-0.63$.

Moreover, on the basis of length characteristics in Cosserat elasticity, the values of the elastic constants required to describe an isotropic constrained Cosserat elastic solid are $\alpha=$ $28.36 \mathrm{kPa}, \quad \gamma=0.0845 \mathrm{~N}$ and $\varepsilon=0.8885 \mathrm{~N}$ for negative Poisson's ratio foam and $\alpha=4.54 \mathrm{kPa}, \gamma=0.199 \mathrm{~N}$ and $\varepsilon=$ $14.382 \mathrm{~N}$ for positive Poisson's ratio foam.

For numerical calculations, values of constants of the impulse load in equation (14) are chosen as: $\alpha^{*}=1.25, p^{*}=$ $2.89, n^{*}=2$ for the case of $t_{*}=8 ; \alpha^{*}=0.9375, p^{*}=$ $1.64, n^{*}=2$ for the case of $t_{*}=12$ and $\alpha^{*}=0.625, p^{*}=$ $0.7225, n^{*}=2$ for the case of $t_{*}=16$, where $t_{*}$ is a dimensionless paremeter, which characterizing of the impulse duration.

Numeric calculations of dynamic normalised radial stresses at the internal points of the foam media are shown in Figs 4-6 for the case $t_{*}=8,12$ and 16 accordingly. Here, $t_{*}$ is the dimensionless parameter characterised by impulse duration: the period of growth and attenuation of load intensity.

Numeric results are calculated for the four values of the distance $\delta$ between the boundary of the cavity and the internal points of medium (Fig. 3) (Mikulich et al., 2017).

In Figs 4-6, curves 1-4 correspond to the case of $\delta=1.5 R$, $\delta=2.5 R, \delta=5 R$ and $\delta=7.5 R$. Numerical calculations are performed for the case of the radius of cavity cross section $R=$ $\ell_{C}$, where $\ell_{C}=\sqrt{\frac{\gamma+\varepsilon}{2 \alpha}}$ is scale parameter in Cosserat elasticity (Sulym et al., 2018). 


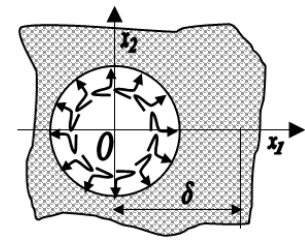

Fig. 3. 2D plane strain geometry of the problem

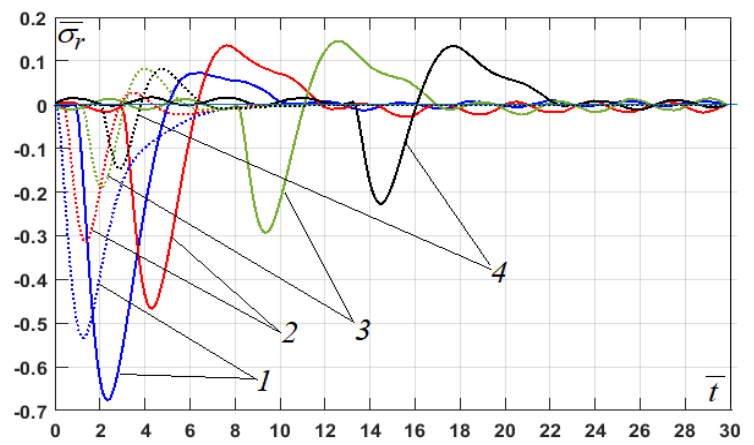

Fig. 4. Distribution of normalised dynamic radial stresses in foam media with tunnel cavity for the case $t_{*}=8$

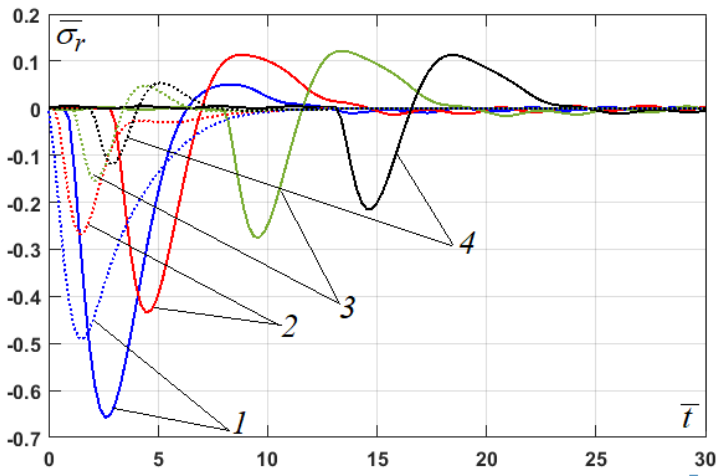

Fig. 5. Distribution of normalised dynamic radial stresses in foam media with tunnel cavity for the case $t_{*}=12$

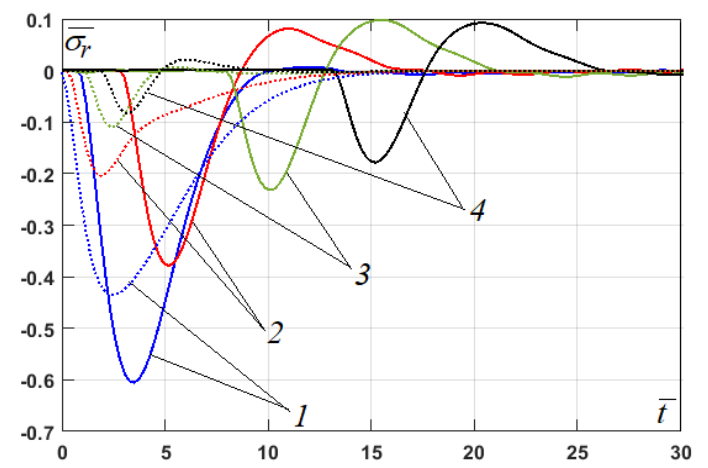

Fig. 6. Distribution of normalised dynamic radial stresses in foam media with tunnel cavity for the case $t_{*}=16$

In Figs 4-6, numeric calculation results of normalised radial stresses are shown by solid lines for the case of negative Poisson's ratio foam and by dotted lines for the case of positive Poisson's ratio foam.

Analysis of results of numerical calculations which is shown in Figs 4-6 confirms that the effect of impulse loading on foam materials causes higher stresses in auxetics than in foam materials with a positive Poisson's ratio. Thus, for the foam materials which are investigated, the maximum values of radial stresses in the interior points of the foam media which are located near the boundary of the cavity are $44 \%-54 \%$ of the intensity applied to the boundary load for the positive Poisson's ratio foam and $60 \%-68 \%$ for negative Poisson's ratio.

However, the speed of wave propagation in the foam medium with negative Poisson's ratio is much lower than in medium with positive Poisson's ratio. It also explains better operational properties of the foam materials with negative Poisson's ratio due to transfer of part of the deformations into sections that are farther away from the defect and to reduce localised effects in the material during its using.

Fig. 7 shows the dependence of the ratio of the maximum values of radial stresses in foam media with a negative and positive Poisson's ratio for changing the distance $\delta$ from the tunnel cavity boundary for different impulse durations.

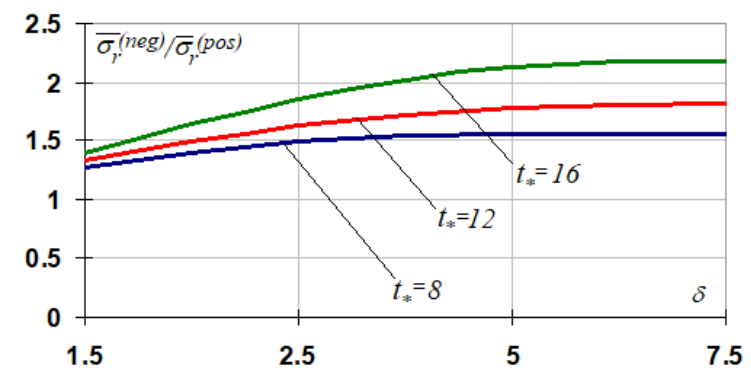

Fig. 7. The ratio of maximum values of radial stresses in foam media

Besides, based on the numerical calculations, it has been shown that for foam auxetic material, the maximum values of normalised dynamic radial stresses are higher by 1.2 to 2.2 times than the corresponding ones calculated for a positive Poisson's ratio foam. It can be explained that auxetic foam materials have a higher density than classical foams. Besides, the load that causes compression deformation in pre-compressed materials passes higher dynamic stresses than in materials with a positive Poisson's ratio.

Also, for foam materials with positive and negative Poisson's ratio, the maximum value of the normalised dynamic radial stresses in the internal points of the stress medium slightly depends on the duration of the impulse. In this case, the smaller the duration of the impulse, the higher are the values of the corresponding stresses. The oscillating character of radial stress distribution in foam materials with negative Poisson's ratio, which occurs after impulse expansion, is explained by lesser stiffness of re-entrant element compared with honeycomb.

\section{CONCLUSION}

Based on modify method of boundary integral equations (Sulym et al., 2018) in the framework of Cosserat continuum, comparison of the distribution of normalised dynamic radial stresses in materials with a positive and negative Poisson's ratio was performed. The results of the numerical calculations confirm the necessity of using refined theories of the continuum mechanics (moment theory of elasticity - Cosserat elasticity) to study the stress state of foam materials with positive and negative Poisson's ratio. 
Based on the numerical calculations, it is established that the distributions of the relative radial stresses in foam media with positive and negative Poisson's ratio have similar characteristics, but the stresses arising from compressive stresses in materials with positive Poisson coefficient are smaller.

An adaptive approach for the investigation of the effect of time variable load on the distribution of radial stresses in foam media can be used to predict the mechanical behaviour of foam auxetic materials obtained at different values of a volumetric compression ratio.

\section{REFERENCES}

1. Brighenti R. (2014), Smart behaviour of layered plates through the use of auxetic materials, Thin-Walled Structures, 84, 432-442.

2. Carneiro V., Meireles J., Puga H. (2013), Auxetic materials - A review, Materials Science-Poland, 31(4), 561-571.

3. Duncan 0., Shepherd T., Moroney Ch., Foster L., Venkatraman Pr, Winwood K., Allen T., Alderson A. (2018), Review of Auxetic Materials for Sports Applications: Expanding Options in Comfort and Protection, Applied Sciences, 8, 941, 1-33.

4. Evans K. (1991), Auxetic Polymers: A New Range of Materials, Endeavour, 15(4), 170-174.

5. Grima J., Attard D., Gatt R., Cassar R. (2009), A Novel Process for the Manufacture of Auxetic Foams and for Their re-Conversion to Conventional Form, Advanced Engineering Materials, 11(7), 533535.

6. Lakes R. S. (1991), Experimental Micro Mechanics Methods for Conventional and Negative Poisson's Ratio Cellular Solids as Cosserat Continua, Journal of Engineering Materials and Technology, 113, 148-155.

7. Lakes R. S. (2016), Physical Meaning of Elastic Constants in Cosserat, Void, and Microstretch Elasticity, Journal of Mechanics of Materials and Structues, 11(3), 217-229.

8. Li D., Dong L., Lakes R. (2016), A Unit Cell Structure with Tunable Poisson's Ratio from Positive to Negative, Materials Letters, 164, 456-459.
9. Mikulich O., Shvabyuk V., Sulym H. (2017), Dynamic Stress Concentration at the Boundary of an Incision at the Plate under the Action of Weak Shock Waves, Acta Mechanica et Automatica, Vol. 11, No. 3, 217-221.

10. Naik S., Dandagwhal R., Wani C., Giri S. (2019), A review on various aspects of auxetic materials. AIP Conference Proceedings, 2105 (1), 10.1063/1.5100689.

11. Novak N., Vesenjak M., Ren Z. (2016), Auxetic Cellular Materials a Review. Journal of Mechanical Engineering, 62(9), 485-493.

12. Nowacki W. (1974), The Linear Theory of Micropolar Elasticity, Springer, New York.

13. Ren X. , Das R., Tran P., Ngo T., Xie Y. (2018), Auxetic Metamaterials and Structures: A Review, Smart Mater. Struct., 27, 1-38.

14. Rueger Z., Lakes R.S. (2016), Cosserat elasticity of negative Poisson's ratio foam: Experiment, Smart Materials and Structures, Vol. 25, 1-8.

15. Scarpa F., Alderson A., Ruzzene M., K. (2016), Auxetics in smart systems and structures, Smart Materials and Structures, 25(5), 1-8.

16. Strek T., Michalski J., Jopek H. (2019) Computational analysis of the mechanical impedance of the sandwich beam with auxetic metal foam core, Physica Status Solidi B, Vol. $256(1), 1800423$ 10.1002/pssb.201800423.

17. Sulym H., Mikulich O., Shvabyuk V. (2018), Investigation of the dynamic stress state of foam media in Cosserat elasticity, Mechanics and Mechanical Engineering, Vol. 22, No.3, 739-750.

18. Underhill R.S. (2017), Manufacture and characterization of auxetic foams, DRDC-RDDC-2017-R099.

19. Zhang X., Ding H., An Li. (2014), Numerical Investigation on Dynamic Crushing Behavior of Auxetic Honeycombs with Various Cell-Wall Angles, Advances in Mechanical Engineering, 10.1155/2014/679678. 